\title{
Active Surface Approach for Extraction of the Human Cerebral Cortex from MRI
}

\author{
Simon F. Eskildsen and Lasse R. Østergaard \\ Dept. of Health Science and Technology, Aalborg University, Denmark
}

\begin{abstract}
Segmentation of the human cerebral cortex from MRI has been subject of much attention during the last decade. Methods based on active surfaces for representing and extracting the cortical boundaries have shown promising results. We present an active surface method, that extracts the inner and outer cortical boundaries using a combination of different vector fields and a local weighting method based on the intrinsic properties of the deforming surface. Our active surface model deforms polygonal meshes to fit the boundaries of the cerebral cortex using a force balancing scheme. As a result of the local weighting strategy and a self-intersection constraint, the method is capable of modelling tight sulci where the image edge is missing or obscured. The performance of the method is evaluated using both real and simulated MRI data.
\end{abstract}

\section{Introduction}

During the last decade, several methods for extracting the boundaries of the human cerebral cortex from magnetic resonance imaging (MRI) have been proposed $[1|2| 3|4| 5|6| 7 \mid 8$. The segmentation of the cerebral cortex may facilitate extraction of important anatomical features, such as the cortical thickness, which may be utilised in studying the progress of a long list of neurodegenerative diseases, and in turn may aid in diagnosing these diseases. Furthermore, anatomical models of the cortex may be useful in connection with surgery simulation, preoperative planning, and postoperative evaluation.

The human cerebral cortex is a complex, highly convolved sheet-like structure. In MRI the cortical boundaries are often obscured or partly missing because of poor contrast, noise, inhomogeneity artifacts and partial volume averaging originating from the acquisition. Opposite banks of tight sulci on the outer boundary may meet inside the sulcal folds and appear as connected in MRI. Active surfaces have the ability to compensate for obscured and incomplete image edges. However, in brain MRI, information of the outer cortical boundary may be completely missing in several tight sulci. The most promising methods for delineating the outer boundary use information of the white matter/grey matter (WM/GM) boundary to fit the surface to the outer cortical boundary. MacDonald et al. used a coupled surface approach, where the inner and outer surface simultaneously were deformed under proximity constraints maintaining a predefined minimum and maximum distance between the inner and outer boundary [6. Zeng et al. also used the coupled surfaces approach in a level set framework [3]. The coupled surfaces approach has the advantage of explicitly using information of both 
cortical boundaries to detect the outer boundary. This solves the problem of penetrating the deep narrow sulci. The drawbacks are the computational expense, and the constraints of a predefined distance, which may prevent the detection of abnormal thin or thick areas of the cortex. Kim et al. proposed a modification to the method by MacDonald et al. which does not contain a coupled surface constraint 8]. This method has shown promising results.

Another approach by Dale et al. identified the inner cortical boundary, and expanded this surface towards the outer boundary [4. This has the advantage that all sulci are present in the initial state, and enables the preservation of the sulci during deformation, even though the evidence of the outer boundary may be missing in the MRI data. The tight sulcal folds are modelled by preventing self-intersections in the deforming surface, thus the delineation of the folds is placed equidistant from the sulcal walls of the inner boundary. This single surface approach is fast and captures all the tight sulci. However, the expansion of the surface towards the outer boundary is sensitive to small errors or irregularities in the initial surface, which may lead to modelling of non-existent sulci. Xu et al. used a Generalised Gradient Vector Flow (GGVF) to define a direction toward the central layer of the cortex [2]. This solution provided a fast and consistently convergence of the surface, but tight sulci with no evidence of the outer boundary were not captured by this method. Recent work by Han et al. expands the surface from the central layer toward the outer boundary using a topology-preserving geometric deformable model [7]. In this approach the GGVF is only included in the model when reconstructing the central cortical layer.

This paper presents an active surface approach for cortex extraction characterised by the inclusion of a GGVF in the extraction of the outer cortical boundary and the use of a local weighting strategy based on the intrinsic properties of the deforming surface.

\section{Methods}

The strategy for reconstructing the cerebral cortex is to first extract the inner boundary, and then displace this surface towards the outer boundary under the influence of internal and external forces. The inner boundary is extracted using the method disclosed in our earlier work 9] ensuring a surface topology of a sphere. The following explains the deformation that fits a surface to the outer cortical boundary using a surface estimating the inner boundary.

\subsection{Deformation Process}

The active surface is a non-parametric triangular mesh. The surface is deformed by iteratively updating each vertex with a vector defined as the sum of deformation forces. This deformation scheme has the advantage of being fast $(O(n))$ and eliminates problems regarding granularity, which is found in discrete methods. Even though convergence may be fast, absolute equilibrium is never reached, due to the iterative nature of the algorithm. Therefore, a threshold for the update vector is given that defines whether or not a vertex has moved during an 
iteration. The stop criterion is met when a sufficiently small number vertices are displaced during an iteration.

During surface deformation the surface is remeshed at prespecified intervals using a simple mesh adaption algorithm. The remeshing is based on the vertex density of the surface. This is done to avoid clustering of vertices and allowing the surface to expand where necessary, i.e. the distribution of vertices are kept uniform throughout the surface. The surface remeshing algorithm does not change the topology of the surface, but is allowed to alter the surface geometry. Finally, the surface is prevented from self-intersecting during deformation using the same principle as described in [4].

\subsection{Internal Forces}

Internal forces are applied to keep the vertices well-distributed and achieve a smooth characteristic of the surface. The internal forces used in this paper are similar to conventional smoothing forces [15] in form of a tensile and a flexural force. The tensile force is calculated by an approximation of the Laplacian [5]:

$$
\boldsymbol{L}(i)=\frac{1}{m} \sum_{j \in N(i)} \boldsymbol{x}(j)-\boldsymbol{x}(i),
$$

where $\boldsymbol{x}(i)$ is the position of vertex $i, N(i)$ are the neighbour vertices to $i, \boldsymbol{x}(j)$ is the position of $i$ 's neighbour $j$, and $m$ is the number of vertices in $N(i)$. The flexural force is calculated by an approximation of the squared Laplacian [5]:

$$
\boldsymbol{L}^{2}(i)=\frac{1}{m} \sum_{j \in N(i)} \boldsymbol{L}(\boldsymbol{x}(j))-\boldsymbol{L}(\boldsymbol{x}(i))
$$

Both $\boldsymbol{L}$ and $\boldsymbol{L}^{\mathbf{2}}$ are decomposed into a tangential and a normal component of the force vector as in the method of Dale et al.[4]. This enables adjustment of the contractive effect of the internal forces by weighting each component.

The internal forces have the effect of smoothing and flattening the surface, however, as the target boundary is highly convolved with both peaked and flat areas, the internal forces should be relaxed in certain areas of the surface and increased in others. The deforming surface is used as a reference for the curvature of the target boundary to obtain local curvature weighting of the internal forces. To enable the surface to compensate for errors in the initial surface, and facilitate some degree of surface curvature alteration, the curvature values are recalculated at prespecified intervals during the deformation process. The curvature is estimated at each vertex of the deforming surface using the expression:

$$
\begin{gathered}
\rho(i)=\left\{\begin{array}{l}
\sigma(i), \text { if } \boldsymbol{w}(i) \cdot \boldsymbol{n}(i) \leq 0 \\
-\sigma(i), \text { otherwise }
\end{array}\right. \\
\sigma(i)=\frac{1}{m} \sum_{j \in N_{g}(i)} \pi-2 \cos ^{-1}\left(\frac{\boldsymbol{x}(j)-\boldsymbol{x}(i)}{|\boldsymbol{x}(j)-\boldsymbol{x}(i)|} \cdot \boldsymbol{w}(i)\right),
\end{gathered}
$$


where $N_{g}(i)$ is a geodesic neighbourhood around vertex $i, \boldsymbol{w}(i)$ is a unit vector pointing from $i$ towards the centre of gravity of $N_{g}(i), \boldsymbol{n}(i)$ is the unit vector normal at $i$, and $m$ is the number of vertices in $N_{g}(i)$. Curvature values of zero are found in flat areas, positive values in convex areas and negative values in concave areas. Note that the size of $N_{g}$ has great influence on the curvature values and should be chosen carefully. The curvature values are Gaussian filtered $(\sigma=1)$, normalised, and in this form used to weight the internal forces:

$$
\hat{\boldsymbol{u}}_{\text {int }}(i)=f(\rho(i)) \boldsymbol{u}_{\text {int }}(i),
$$

where $f$ is a weighting function defined as

$$
f(x)=1-\frac{1}{2} \tan (|x|), x \in[-1 ; 1]
$$

\subsection{External Forces}

The outer boundary of the cerebral cortex follows approximately the same convexities and concavities as the inner cortical boundary. Hence, a surface delineating the inner cortical boundary is used as an initial estimate of the outer cortical boundary. This inner surface is displaced in the direction of the local surface normals until the surface meets itself (see figure 1), and thereby model sulci, even though only little or no image information is available. For this purpose a pressure force [1] is used. The force is similar to the external force used in [5]4, but based on fuzzy memberships of the tissue classes as described in [2]. The fuzzy memberships are calculated using the fuzzy c-means algorithm [10. The pressure force is expressed as:

$$
\begin{aligned}
& \boldsymbol{p}(i)=\Delta \mu(i) \boldsymbol{n}(i) \\
& \Delta \mu(i)=\mu_{W M}(i)+\mu_{G M}(i)-\mu_{C S F}(i),
\end{aligned}
$$

where $\mu$ is the membership values (trilinearly interpolated) and $\boldsymbol{n}(i)$ is the unit vector normal at vertex $i$. A weighting function is applied to the membership difference to ensure a degree of freedom at membership differences close to zero:

$$
\hat{\boldsymbol{p}}(i)=c_{1} g(\Delta \mu(i)) \boldsymbol{n}(i),
$$

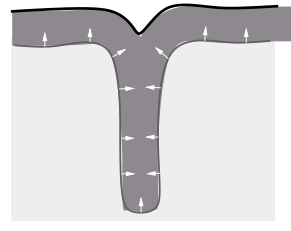

(a) Initial

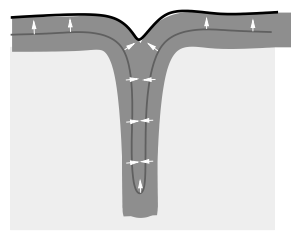

(b) Deforming

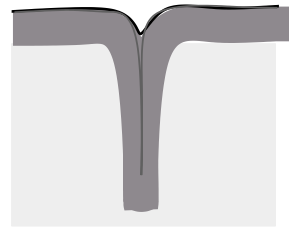

(c) Final

Fig. 1. Illustration of how the pressure force enables modelling of narrow sulci with no CSF evident. As the deformable surface (grey line) is pushed away from the WM/GM boundary in the direction of the local surface normals, it will eventually meet itself inside narrow sulci. 
where $c_{1}$ is a weighting constant and

$$
g(x)=x(2-2 \cos (x)), x \in[-1: 1]
$$

Surface normals, approximated from a discrete mesh, may be misleading, as they can be perturbed by noise in the surface. This may erroneously cause modelling of non-existing features, when the surface is displaced over larger distances. Increasing the influence of the internal forces resolves this problem, but also prevents the surface from reaching small concavities, which are truly evident in the MRI. To solve the problem, the pressure force is combined with a GGVF force similar to the one used by $\mathrm{Xu}$ et al. 2], but with an edge map of the outer cortical boundary instead of the central cortical layer. This edge map is the first order derivative of the sum of the WM and GM fuzzy memberships. The GGVF force performs best at the gyri where information of the GM/CSF boundary is evident in the MRI, thus the normal vector is combined with the GGVF vector so the GGVF vector dominates the normal vector at the crown and ridges of gyri, and the normal vector dominates the GGVF vector along the fundi, and walls of sulci. The local surface curvature, calculated in a geodesic neighbourhood, is used for balancing the influence of the GGVF vector and the normal vector:

$$
\boldsymbol{u}_{e x t}(i)=c_{2}\left(\hat{\boldsymbol{p}}(i) \frac{1-\rho(i)}{2}+\boldsymbol{g}(i) \frac{1+\rho(i)}{2}\right),
$$

where $\hat{\boldsymbol{p}}(i)$ is the pressure force vector at vertex $i, \boldsymbol{g}(i)$ is the GGVF vector at vertex $i, \rho(i)$ is the curvature value at $i$ given in (3), and $c_{2}$ is a constant.

Gradient information is used to scale the update vector $\boldsymbol{u}$, so the magnitude of the update vector is reduced when the magnitude of the gradient increases. This is done by mapping the normalised gradient magnitudes with the function:

$$
h(x)=\cos \left(\frac{\pi}{2} x\right), x \in[0: 1]
$$

and scaling the update vector $\boldsymbol{u}$ by the result. The update vector is unchanged when there is no gradient and greatly shortened when a strong gradient is present at the given vertex position. Information of the gradient is used only when close to the GM/CSF boundary and suppressed when far from the boundary. The weighted membership difference $g(\Delta \mu(i))$ in (8), that provides an estimate for the spatial position of the GM/CSF boundary, is therefore utilised to weight the influence of the gradient. The resulting update vector is given as a weighted sum of a gradient weighted term and a non-gradient weighted term:

$$
\begin{aligned}
& \boldsymbol{u}(i)=\left((1-\tau) \cos \left(\frac{\pi}{2}|\nabla(i)|\right)+\tau\right)\left(\hat{\boldsymbol{u}}_{\text {int }}(i)+\boldsymbol{u}_{\text {ext }}(i)\right), \\
& \tau=|g(\Delta \mu(i))|
\end{aligned}
$$

where $\boldsymbol{\nabla}(i)$ is the image gradient trilinearly interpolated at vertex $i, \hat{\boldsymbol{u}}_{\text {int }}(i)$ is the weighted sum of the internal forces given in (5), and $\Delta \mu(i)$ is given in (7). 


\section{Results}

Simulated MRI scans of a brain phantom 11 and 36 T1 weighted MRI datasets of young normal subjects from the International Consortium for Brain Mapping (ICBM) database were used for testing the general behaviour of the deformation. The same weighting constants were used in all test cases. Initial surfaces isomorph to a sphere were generated and fitted to the inner cortical boundary. The initial surfaces consisted of approximately $1.5 \cdot 10^{5}$ vertices. During deformation this number was increased to approximately $2.0 \cdot 10^{5}$. The deformation process converged after 30-40 iterations with the stop criterion of (\#moved vertices $)<(1 \%$ of total vertices $)$. The deformation of the outer surface required approximately 20 minutes on a $3 \mathrm{GHz}$ Pentium 4 processor. The self-intersection tests performed throughout the deformation of the inner and outer surface were responsible for the majority of the processing time.

Figure 2 shows three different modes of the deformation process in a selected part of the simulated MRI. The three modes differ in their external forces, the internal forces are the same for all three modes. In the first mode, only the



Fig. 2. Outer surface deformation process using different external forces at different stages in the process. Left to right: Deformation process at iterations 0,5,15 and 30 . Top: Only pressure force is enabled. Middle: Only GGVF force is enabled. Bottom: Combination of both forces balanced by the curvature weighting function.

${ }^{1}$ The brain phantom was provided by the McConnel Brain Imaging Centre at the Montreal Neurological Institute, http://www.bic.mni.mcgill.ca 


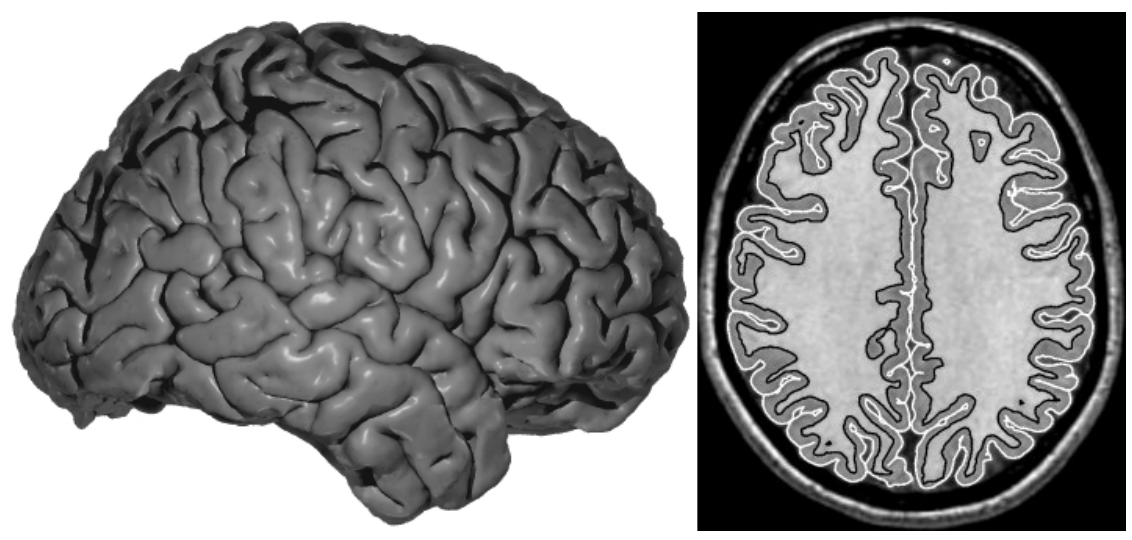

Fig. 3. Example of a generated cortex from ICBM data. Left: Rendering of outer surface. Right: Inner (black) and outer (white) surfaces superimposed onto MRI.

pressure force is enabled, simulating the method by Dale et al. 4]. This clearly shows that the use of the pressure force alone result in irregularities in the surface. This is especially evident at top of gyri. In the second mode, only the GGVF force is enabled, simulating the method by Xu et al. [2]. In this case the surface does not reach the fundus of sulci without evidence of CSF. There is also an undesirable behaviour in some of the sulci, because the surface is attracted to the nearest visible GM/CSF image edge. The last mode shows deformation with both the pressure force and the GGVF force enabled, balanced using the curvature weighting function. Now the tight sulci are being modelled correctly while avoiding surface irregularities on top of gyri.

All 36 cortices from the ICBM database were automatically reconstructed and qualitatively assessed by visual inspection. An example of an extracted outer surface is shown in figure 3, As it can be seen from the figure, the extracted surface appears smooth, realistic and major gyri and sulci are easily recognised. The qualitative assessment of the accuracy of the extracted surfaces was made by superimposing the surfaces onto the MRI and visually inspecting the contours (figure 3, right). As it can be observed from the figure the outer cortical boundary is accurately delineated. Tight sulci are modelled even when the crowns of adjacent gyri are not separated in the image data, and the surface tend to be placed at a position equidistant to the WM walls when no CSF is evident in sulci. This indicates that the method follows the intended behaviour.

\section{Summary and Conclusion}

This paper presented a new method for extracting the outer boundary of the human cerebral cortex from MRI. The active surface approach combines a conventional pressure force with fuzzy tissue classifications, and a generalised gradient vector flow force, while locally weighting the forces based on the surface 
curvature. Preliminary tests were conducted on both simulated data and real data of young normal subjects. The primary results of these tests indicate that the method is fast, robust and accurate for segmenting the cortical boundary in both simulated and real neuroanatomical data. Still, the method needs further validation, as it must be able to perform on data with varying quality and from a varying population, if it is going to be applicable in everyday clinical use. Future work include a large scale validation on both healthy subjects and subjects with altered cortical morphology.

\section{Acknowledgements}

Test data were provided courtesy of the International Consortium of Brain Mapping and McConnell Brain Imaging Centre, Montreal Neurological Institute, McGill University.

\section{References}

1. Cohen, L.D., Cohen, I.: Finite-element methods for active contour models and balloons for 2D and 3D images. IEEE Trans. Pattern Analysis and Machine Intelligence (1993)

2. Xu, C., Pham, D.L., Rettmann, M.E., Yu, D.N., Prince, J.L.: Reconstruction of the human cerebral cortex from magnetic resonance images. IEEE Trans. Medical Imaging 18(6) (1999) 467-480

3. Zeng, X., Staib, L.H., Schultz, R.T., Duncan, J.S.: Segmentation and measurement of the cortex from 3-d mr images using coupled-surfaces propagation. IEEE Trans. Medical Imaging 18(10) (1999) 100-111

4. Dale, A.M., Fischl, B., Sereno, M.I.: Cortical surface-based analysis i: Segmentation and surface reconstruction. NeuroImage 9 (1999) 179-194

5. McInerney, T., Terzopoulos, D.: Topology adaptive deformable surfaces for medical image volume segmentation. IEEE Trans. Medical Imaging 18(10) (1999) 840-850

6. MacDonald, D., Kabani, N., Avis, D., Evans, A.C.: Automated 3-D extraction of inner and outer suraces of cerebral cortex from mri. NeuroImage 12 (2000) 340-356

7. Han, X., Pham, D., Tosun, D., Rettmann, M., Xu, C., Prince, J.: Cruise: Cortical reconstruction using implicit surface evolution. NeuroImage 23(3) (2004) 997-1012

8. Kim, J.S., Singh, V., Lee, J.K., Lerch, J., Ad-Dab'bagh, Y., MacDonald, D., Lee, J.M., Kim, S.I., Evans, A.C.: Automated 3-d extraction and evaluation of the inner and outer cortical surfaces using a laplacian map and partial volume effect classification. NeuroImage 27(1) (2005) 210-221

9. Eskildsen, S.F., Uldahl, M., Østergaard, L.R.: Extraction of the cerebral cortical boundaries from mri for measurement of cortical thickness. Progress in Biomedical Optics and Imaging - Proceedings of SPIE 5747(2) (2005) 1400-1410

10. Pham, D., Prince, J.: Adaptive fuzzy segmentation of magnetic resonance images. IEEE Transactions on Medical Imaging 18(9) (1999) 737-752

11. Collins, D.L., Zijdenbos, A.P., Kollokian, V., Sled, J., Kabani, N., Holmes, C., Evans, A.: Design and construction of a realistic digital brain phantom. IEEE Transactions on Medical Imaging 17(3) (1998) 463-468 